\title{
Peliosis Hepatis-The Chameleon: An Unusual Presentation Mimicking Liver Abscess
}

\author{
Ruchi Rastogi ${ }^{1}$ Parveen Batra ${ }^{1} \quad$ Subhash Gupta ${ }^{1} \quad$ Anjali K. Gupta ${ }^{1} \quad$ Bharat Aggarwal ${ }^{1} \quad$ Shaleen Agarwal ${ }^{1}$ \\ ${ }^{1}$ Department of Radiology, Max Super Speciality Hospital, New \\ Delhi, India

\begin{abstract}
Address for correspondence Ruchi Rastogi, MD, E 243, Third Floor, Greater Kailash II, New Delhi 110048, India

(e-mail: drruchirastogi@yahoo.com).
\end{abstract}

J Gastrointestinal Abdominal Radiol ISGAR 2019;2:126-129

\begin{abstract}
Keywords

- peliosis hepatis

- liver abscess

- abdominal computed tomography

- abdominal magnetic resonance imaging

Peliosis hepatis is a rare vascular condition characterized by multiple randomly distributed blood-filled cavities throughout the liver, ranging in size from a few millimeters to several centimeters in diameter. The appearance of peliotic lesion on imaging varies depending on morphologic type, concomitant hemorrhagic component, and presence or absence of background hepatic steatosis. Here, we report an unusual presentation of peliosis hepatis as an exophytic mass arising in liver parenchyma, mimicking a liver abscess.
\end{abstract}

\section{Introduction}

The term "peliosis" originated from a Greek word "pelios," which means "purple," referring to the color of affected liver with peliosis. It was first described by Wagner in 1861 and was later named by Schoenlank in 1916. Yanoff and Rawson ${ }^{1}$ described two morphologic patterns of peliosis, phlebectatic, and parenchymal type. The genesis of peliosis hepatis is multifactorial, including use of various drugs (e.g., anabolic steroids, oral contraceptives, corticosteroids, diethylstilbestrol, 6-thioguanine, 6-mercaptopurine, methotrexate, tamoxifen, and azathioprine); consumption of toxins (e.g., polyvinyl chloride, arsenic, and thorium oxide); chronic illness (e.g., tuberculosis, leprosy, celiac sprue); diabetes mellitus, necrotizing vasculitis; hematologic disorders (Hodgkin's disease, multiple myeloma, and various malignancies); and infection in AIDS (known as bacillary peliosis caused by Bartonella henselae and B. quintana). In addition, peliosis hepatis may develop after renal or cardiac transplantation. However, the etiology is idiopathic in up to $50 \%$ of the patients.

\section{Clinical Presentation with Radiologic and Pathologic Workup}

A 30-year-old woman was referred to our hospital with complaint of abdominal bloating for 1 month. She had a history of sudden-onset abdominal pain and was hospitalized in an

received

November 28, 2018 accepted after revision

March 26, 2019

published online

July 31, 2019
African country, where she was treated with analgesics and discharged. She improved symptomatically, but due to persistent abdominal discomfort, she underwent ultrasound and was diagnosed to have a large cystic hepatic lesion. Ultrasound (USG)-guided biopsy was done to evaluate the lesion, which revealed presence of inflammatory infiltrates with absence of any mitotic cells, suggesting a possibility of liver abscess. Noncontrast computed tomographic (NCCT) scan and a single-phase contrast-enhanced magnetic reson-ance imaging (MRI) of the abdomen in the venous phase were obtained subsequently for the same. Axial NCCT images revealed large hypodense lesion arising from the left lobe of the liver with presence of nondependant air loculi and air-fluid level ( - Fig. 1A). The single-phase contrast-enhanced MRI scans showed a large peripherally enhancing exophytic mass lesion arising from left lobe of the liver ( - Fig. 1B and C). The periphery of the mass was thick and irregular with heterogenous postcontrast enhancement. No definite lesion could be seen in the remaining liver parenchyma in this scan.

The patient was referred for further management at our hospital. Her vital signs showed a blood pressure of $140 / 86 \mathrm{~mm} \mathrm{Hg}$, a heart rate of 82 beats/min, a respiratory rate of 18 breaths $/ \mathrm{min}$, and a temperature of $39^{\circ} \mathrm{C}$. Physical examination revealed a firm, nontender liver edge palpable $2 \mathrm{~cm}$ below the right costal margin. Examination revealed no other findings such as jaundice, cutaneous stigmata of chronic liver disease, ascites, or splenomegaly. Laboratory examination showed white blood cell count of $12 \times 10^{9} / \mathrm{L}$
(C)2019 Indian Society of

Gastrointestinal and Abdominal Radiology
License terms

10.1055/s-0039-1692020

ISSN 2581-9933.

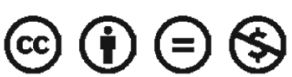




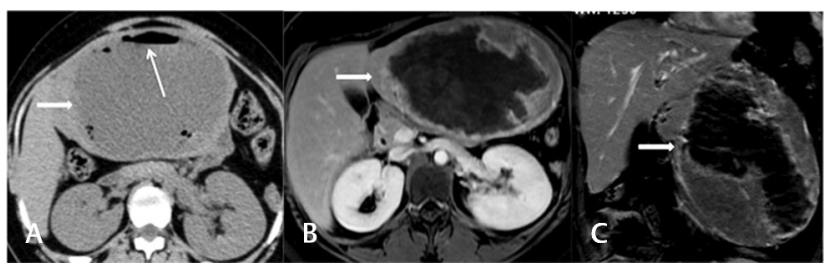

Fig. 1 (A) Axial unenhanced computed tomography image shows a large hypodense exophytic lesion arising from left lobe of the liver, with fluid attenuation at center and few air loculi in it. A small air-fluid level is seen in nondependent position (thin long arrow). (B, C) Contrast-enhanced axial and coronal fat-suppressed T1-weighted magnetic resonance imaging done outside show presence of a large exophytic lesion arising from left hepatic lobe of the liver (arrow) showing thick peripheral enhancement with central necrotic area.

(normal $4 \times 10^{9}-10 \times 10^{9}$ ), hemoglobin of $8.7 \mathrm{mg} / \mathrm{dL}$ (normal 13.1-17.5 g/L), hematocrit of $29 \%$ (normal $39-52 \%$ ), platelet count of $623 / \mathrm{L}\left(\right.$ normal $\left.150 \times 410 \times 10^{9} / \mathrm{L}\right)$, alkaline phosphatase of $123 \mathrm{IU} / \mathrm{L}$ (normal 32-91 IU/L), alanine aminotransferase of $111 \mathrm{IU} / \mathrm{L}$ (normal 32-91 IU/L), aspartate aminotransferase of $66 \mathrm{IU} / \mathrm{L}$ (normal 14-54 IU/L), and international normalized ration (INR) of 11.4 seconds (normal 11-15 seconds). Serum bilirubin levels including direct, indirect, and total bilirubin were normal. Total protein, albumin level, and albumin-to-globulin ratio were decreased. Serologic markers including HIV1/HIV2 antibodies, hepatitis C IgG antibodies, and HBs antigen levels were within normal limits.

On reviewing the images at our institute, a presumptive diagnosis of benign hypervascular necrotic liver mass was made. Although the histopathologic report of USG-guided biopsy performed previously had suggested the possibility of liver abscess, the enhancement characteristics on MRI with large peripheral enhancing solid component did not support liver abscess as the primary diagnosis. The presence of air-fluid level in lesion could also be explained by history of previous intervention, besides the possibility of infective etiology of lesion. Considering discordance between imaging and biopsy findings and persistence of lesion and clinical complaints, a nonanatomical left lobe resection was performed.

Pathologic examination of the resected specimen showed a large cavitary lesion measuring $15 \times 10 \times 12 \mathrm{~cm}$ in size. Inner surface showed irregular, gray-brown nodularity along with necrotic friable material ( $\boldsymbol{- F i g .} \mathbf{3 A}$ and $\mathbf{B}$ ). Multiple sections examined from the wall of the large cavity in the liver showed an acute abscess wall cordoned off from the liver by a thick wall lined by hemosiderin-laden macrophages ( - Fig. $\mathbf{3 C}-\mathbf{E}$ ). The luminal contents were necroinflammatory in nature and bacterial colonies were noted. No histopathologic evidence of hepatic adenoma or hepatocellular carcinoma was present. Considering a suspicion of liver abscess on USG-guided biopsy and previous CT scan, it was a diagnostic dilemma for the pathologist.

However, in view of presence of thick peripheral heterogenous enhancement on MRI, the pathologist was requested to have a relook at the periphery of the mass.

On further evaluation, the surrounding liver was found to show multiple areas of peliosis ( $\mathbf{- F i g}$. $\mathbf{3 F}$ ). The parenchyma surrounding these areas of peliosis showed hepatocyte plate atrophy. Macrovesicular steatosis was noted (45\%) along with focal cholestasis. No parasitic trophozoite, granuloma, and dysplastic or malignant cells were seen. Presence of bacterial colonies in the walls of necrotic exophytic lesion could be related to the bacterial invasion during biopsy or spontaneous hematologic spread.

A contrast-enhanced MRI was obtained in the postoperative period in view of histopathologic findings on a 3.0 Tesla scanner. It included axial and coronal T2-weighted turbo spin echo sequences, axial dual-echo T1-weighted in-opposed phase sequence, and diffusion-weighted images using b values of 0 and 800 milliseconds. Fat-suppressed three-dimensional (3D) T1-weighted gradient recalled echo (GRE) sequences were performed in axial plane before and after intravenous injection of gadolinium dimeglumine (Multihance, Bracco). It showed postsurgical changes and multiple subcentimetric sized lesions showing arterial phase enhancement in liver parenchyma ( - Fig. 2A-C). Most lesions were isointense to surrounding liver parenchyma on venous phases, with few of the lesions showing mild hypoenhancement. Few of these lesions showed mildly restricted diffusion ( - Fig. 2D). These small lesions were not well visualized on routine $\mathrm{T} 1$ - and $\mathrm{T} 2$-weighted images.

The sum of clinical, imaging, and pathologic findings supported the diagnosis of peliosis hepatis, with superimposed infection in an old hemorrhagic peliotic lesion in left lobe of the liver.

\section{Discussion}

The classic pathoanatomic concept for development of peliotic lesions is based on the opinion that peliosis exclusively develops in organs belonging to the mononuclear phagocytic system (liver, spleen, bone marrow, and lymph nodes). However, other organs such as lungs, parathyroid glands, and kidneys may be affected too. As far as the pathogenesis is

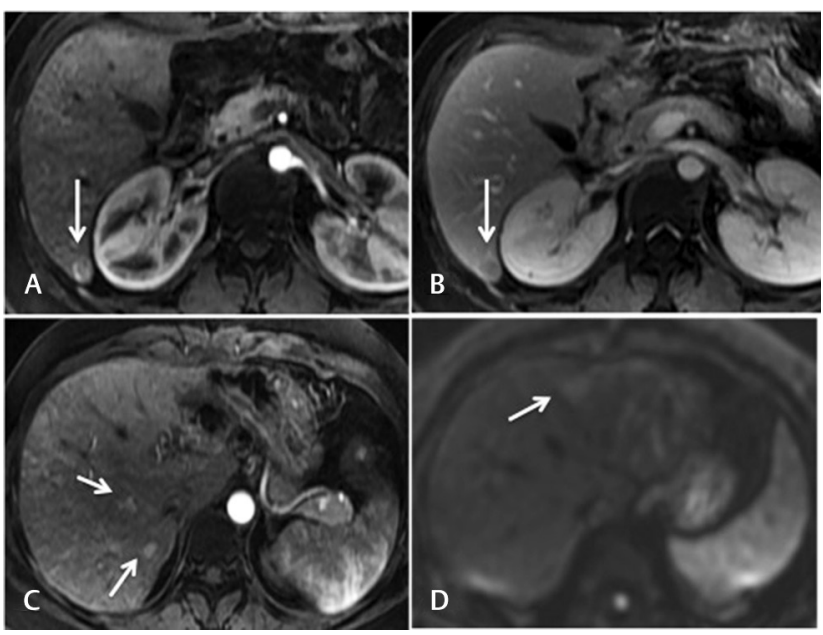

Fig. 2 Multiphasic contrast magnetic resonance imaging done in postoperative period. Fat-suppressed T1W images (A and $\mathbf{B}$ ) reveal presence of a small nodule in segment 6 of the liver showing arterial phase enhancement (arrow, A), followed by hypoenhancement on venous phase (arrow, B). T1W arterial phase image at a higher level (C) demonstrates presence of multiple other small arterial enhancing foci in remaining liver parenchyma (arrows). Most of these nodules were becoming isointense on subsequent venous and equilibrium phases (not shown here). Few of the nodules show mild diffusion restriction (arrow, D). 


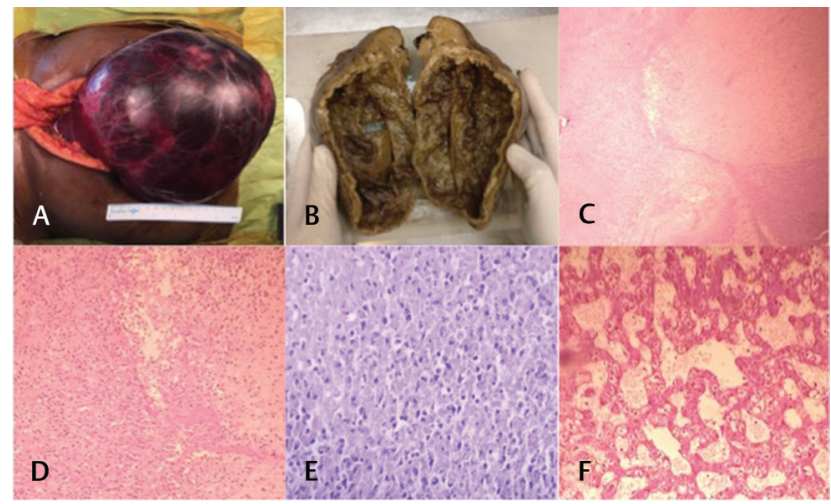

Fig. 3 Intraoperative photograph of the exophytic mass projecting out of the abdominal cavity through the surgical incision shows the classic purple color with smooth surface (A). The cut section of gross specimen shows central cavity with thick periphery (B). Hematoxylin and eosin (H\&E) staining at $10 \times$ magnification shows wall of abscess cavity with wall above and inflammatory infiltration below (C). Same area is shown at a higher magnification (D). H\&E staining at $40 \times$ magnification shows inflammatory cells in the abscess cavity (E). Areas of peliosis are seen at the margins of lesion $(\mathbf{F})$

concerned, more than one path of formal pathogenesis is proposed. There can be congenital malformation of vessels manifesting under altered local intravascular pressure conditions such as in chronic renal disease or it can be an acquired vascular disorder triggered by toxins like vinyl chloride, arsenic, and thorium oxide. ${ }^{2}$ Various investigators proposed that the primary event could be obstruction of hepatic outflow at the sinusoidal level, direct breakdown of sinusoidal borders, dilatation of the central vein of the hepatic lobule, or hepatocellular necrosis leading to cavity formation. ${ }^{3}$

Clinical presentation of peliosis hepatis may range from an asymptomatic incidentally discovered lesion to a catastrophic presentation due to its hemorrhagic complication. Similarly, the imaging findings are also variable. Multiple factors such as hemorrhagic component, fatty changes, pathological pattern, and calcification give rise to a spectrum of appearances on imaging. The lesions may mimic other hepatic masses, such as hemangioma, hepatocellular carcinoma, abscess, metastasis, adenoma, and focal nodular hyperplasia. ${ }^{4}$

\section{Sonographic Findings}

The appearance of peliotic lesions on gray scale sonography depends on presence or absence of steatosis in liver parenchyma. The lesions appear hyperechoic in healthy liver and hypoechoic in patients with steatotic liver parenchyma. These may be homogenous or heterogenous, depending on presence or absence of necrosis and hemorrhage. Kleinig et $\mathrm{al}^{5}$ demonstrated a transient "fast surge" central echo enhancement in arterial phase in peliotic lesions using sonographic contrast agent. During the portal and sinusoidal phases, peliosis hepatis demonstrates iso- or hypoechogenicity with no contrast material pooling or centripetal filling, allowing differentiation of this entity from hemangioma.

\section{CT Findings}

CT appearance varies from normal to hypo- or hyperdense lesions, depending on their size, presence of intralesional hemorrhage, and calcification. Smaller lesions may not be appreciated on unenhanced scans. The larger lesions typically appear as low-attenuation areas, but the presence of intralesional blood produces hyperattenuating appearance compared with the background liver parenchyma. On contrast study, smaller lesions may show uniform smooth enhancement in both arterial and venous phases. A larger peliotic lesion shows early globular vessel-like areas of enhancement in arterial phase. Sometimes there may be central small accumulation of contrast agent in the arterial phase, called target sign. During the portal venous phase, usually a centrifugal progression of enhancement is observed; however, a centripetal progression of enhancement can also be seen, mimicking hemangioma. On the delayed phase, there is accumulation of contrast in the lesions producing diffuse homogeneous hyperattenuation, especially in the phlebectatic type.

The features such as vessel-like early arterial phase enhancement, centrifugal filling in portovenous phase, and contrast accumulation (central in arterial phase and diffuse in delayed phase) differentiate peliotic lesions from other focal lesions of the liver. ${ }^{6}$

\section{MRI Findings}

Imaging findings on MRI are variable. Depending on hemorrhagic component and age of blood products, lesions show variable signal intensities on T1- and T2-weighted images. T1-weighted images may demonstrate hypo-, iso-, or hyperintense areas within the lesion. On T2-weighted images, the lesions are predominantly hyperintense. The lesions are typically surrounded by hepatic parenchyma, rarely seen extending outside the liver contours. Presence of fluid-fluid levels may also be present occasionally. Diffusion-weighted images are particularly helpful in detection of smaller lesions. Although peliosis hepatis is a benign condition, apparent diffusion coefficient (ADC) values may be less than normal-appearing liver, due to its hemorrhagic contents. ${ }^{7}$

\section{Angiographic Findings}

Peliotic lesions are also well demonstrable on conventional angiographic imaging. Pliskin ${ }^{8}$ reported a case showing multiple small areas with contrast material accumulations ranging in size from barely visible to more than $1 \mathrm{~cm}$ in diameter in a peliotic lesion in the late arterial phase of conventional hepatic angiogram that became more prominent on parenchymal and venous phases.

In our case, most lesions showed typical arterial enhancement with iso- to mildly hypointense signal in portovenous phase. The largest lesion involving left lobe showed peripheral enhancement with central necrotic hemorrhagic component. Most lesions were surrounded by hepatic parenchyma, except for the left lobar lesion that was exophytic. Till date only two cases of exophytic peliotic lesions have been reported, by Ferrozzi et $\mathrm{al}^{9}$ and Battal et al, ${ }^{7}$ respectively.

\section{Histopathologic Examination}

Histology is always required to confirm the diagnosis. Microscopically, the parenchymal type of lesion consists of irregular cavities that are lined by neither sinusoidal cells 
nor fibrous tissue. On the other hand, phlebectatic type of lesion is characterized by regular, spherical cavities lined by endothelium and/or fibrosis. In the spleen, the peliotic lesions may be arranged sporadically, disseminated, or in clusters in an uneven distribution pattern. Closest differential diagnoses for these findings include secondary hepatic congestion due to veno-occlusive disease or Budd-Chiari syndrome, splenic hemangiomas, and involvement of the spleen in hairy-cell leukaemia. ${ }^{2}$

\section{Clinical Importance}

Misdiagnosis of this otherwise uncommon entity can lead to disastrous outcomes in selected clinical situations. There is a similar case reported by Cohen et $\mathrm{al}^{10}$ in which a large peliotic lesion was diagnosed as liver abscess and percutaneous catheter drainage was performed. The patient went into shock and an emergent conventional hepatic arteriogram obtained 12 hours after the drainage to detect the site of bleeding revealed multiple 1 - to 3 -mm-sized focal collections of contrast material in the late arterial phase extending well beyond the avascular necrotic cavity seen on previous CT scan. These findings suggested the possibility of a peliotic lesion. Emergency laparotomy was planned, but the patient died of cardiac arrest. Autopsy findings confirmed the diagnosis. This was a fatal outcome of an otherwise benign vascular lesion.

To our knowledge, there is no case report of such a large peliotic exophytic mass with central necrosis and suppurative infection. The role of radiologist is to suspect this obscure diagnosis in situations when imaging findings are atypical. For example, in a case of cystic liver lesion, presence of thick irregular peripheral hyperenhancement, especially in the early arterial phase or bloody rather than purulent aspirate on cyst fluid aspiration, may indicate a possibility of underlying peliosis hepatis, hemangioma, or necrotic and infected tumor. An extra effort should be made to look for additional smaller lesions if peliosis is suspected. Arteriography may also be considered in selected cases as inadvertent biopsies may also sometimes prove fatal.

\section{Conclusion}

Peliosis hepatis must be added to the list of differential diagnosis of a large centrally necrotic and hemorrhagic liver lesion that shows early enhancement, especially if associated with other smaller lesions showing imaging pattern typical of peliosis. The role of diffusion-weighted MRI scans and multiphasic contrast-enhanced cross-sectional study is further emphasized, not to miss small lesions visible only on early arterial phase images and remaining occult in other phases.

This case is an important contribution to the literature on peliosis hepatis, as it is rarely encountered as a large exophytic hepatic mass with central necrotic degeneration and abscess-like presentation.

\section{Funding}

None.

\section{Conflict of Interest}

None declared.

\section{Acknowledgments}

The authors sincerely thank Mr. Lejoy Kuruvilla and Mr. Peter for their technical support.

\section{References}

1 Yanoff M, Rawson AJ. Peliosis hepatis. An anatomic study with demonstration of two varieties. Arch Pathol 1964;77:159-165

2 Tsokos M, Erbersdobler A. Pathology of peliosis. Forensic Sci Int 2005;149(1):25-33

3 Wanless IR. Vascular disorders. In: MacSween RNM, Burt AD, Portmann BC, Ishak KG, Scheuer PJ, Anthony PP, eds. Pathology of the Liver. 4th ed. Glasgow, UK: Churchill Living stone; 2002:553-555

$4 \mathrm{Kim}$ SH, Lee JM, Kim WH, Han JK, Lee JY, Choi BI. Focal peliosis hepatis as a mimicker of hepatic tumors: radiological-pathological correlation. J Comput Assist Tomogr 2007;31(1):79-85

5 Kleinig P, Davies RP, Maddern G, Kew J. Peliosis hepatis: central "fast surge" ultrasound enhancement and multislice CT appearances. Clin Radiol 2003;58(12):995-998

6 Iannaccone R, Federle MP, Brancatelli G, et al. Peliosis hepatis: spectrum of imaging findings. Am J Roentgenol 2006;187(1):W43-W52

7 Battal B, Kocaoglu M, Atay AA, Bulakbasi N. Multifocal peliosis hepatis: MR and diffusion-weighted MR-imaging findings of an atypical case. Ups J Med Sci 2010;115(2):153-156

8 Pliskin M. Peliosis hepatitis. Radiology 1975;114(1):29-30

9 Ferrozzi F, Tognini G, Zuccoli G, Cademartiri F, Pavone P. Peliosis hepatis with pseudotumoral and hemorrhagic evolution: CT and MR findings. Abdom Imaging 2001;26(2): 197-199

10 Cohen GS, Ball DS, Boyd-Kranis R, Gembala RB, Wurzel J. Peliosis hepatis mimicking hepatic abscess: fatal outcome following percutaneous drainage. J Vasc Interv Radiol 1994;5(4):643-645 